\title{
Prevalence and Predictors of Night Sweats, Day Sweats, and Hot Flashes in Older Primary Care Patients: An OKPRN Study
}

James W. Mold, MD, MPH

Michelle Roberts, $B A^{2}$

Hesham M. Aboshady, MD, ChB, $M P H^{3}$

${ }^{1}$ Oklahoma Center for Family Medicine Research, Department of Family and Preventive Medicine, University of Oklahoma Health Sciences Center, Oklahoma City, Okla

${ }^{2}$ University of Oklahoma Health Sciences Center, Oklahoma City, Okla

${ }^{3}$ Private practice, North Providence, RI

\begin{abstract}
PURPOSE We wanted to estimate the prevalence of night sweats, day sweats, and hot flashes in older primary care patients and identify associated factors.
\end{abstract}

METHODS We undertook a cross-sectional study of patients older than 64 years recruited from the practices of 23 family physicians. Variables included sociodemographic information, health habits, chronic medical problems, symptoms, quality of life, and the degree to which patients were bothered by night sweats, daytime sweating, and hot flashes.

RESULTS Among the 795 patients, 10\% reported being bothered by night sweats, $9 \%$ by day sweats, and $8 \%$ by hot flashes. Eighteen percent reported at least 1 of these symptoms. The 3 symptoms were strongly correlated. Factors associated with night sweats in the multivariate models were age (odds ratio [OR] 0.94/y; 95\% confidence interval [Cl], 0.89-0.98), fever (OR 12.60; $95 \% \mathrm{Cl}$, 6.58-24.14), muscle cramps (OR 2.84; $95 \% \mathrm{Cl}, 1.53-5.24)$, numbness of hands and feet (OR 3.34; 95\% Cl, 1.92-5.81), impaired vision (OR 2.45; 95\% Cl, 1.414.27), and hearing loss (OR $1.84 ; 95 \% \mathrm{Cl}, 1.03-3.27)$. Day sweats were associated with fever (OR 4.10; $95 \% \mathrm{Cl}, 2.14-7.87$ ), restless legs (OR 3.22; $95 \% \mathrm{Cl}, 1.76$ 5.89), lightheadedness (OR 2.24; 95\% Cl, 1.30-3.88), and diabetes (OR 2.19; $95 \% \mathrm{Cl}, 1.22-3.92)$. Hot flashes were associated with nonwhite race (OR 3.10; 95\% Cl, 1.60-5.98), fever (OR 3.98; 95\% Cl, 1.97-8.04), bone pain (OR 2.31; Cl 95\%: 1.30-4.08), impaired vision (OR 2.12; $95 \% \mathrm{Cl}, 1.19-3.79)$, and nervous spells (OR 1.87; $95 \% \mathrm{Cl}, 1.01-3.46)$. All 3 symptoms were associated with reduced quality of life.

CONCLUSION Many older patients are bothered by night sweats, day sweats, and hot flashes. Though these symptoms are similar and related, they have somewhat different associations with other variables. Clinical evaluation should include questions about febrile illnesses, sensory deficits, anxiety, depression, pain, muscle cramps, and restless legs syndrome.

Ann Fam Med 2004;2:391-397. DOI: 10.1370/afm.72.

\section{INTRODUCTION}

$\mathrm{B}$ ecause information from primary care settings is often lacking, primary care physicians often must rely upon information derived from subspecialty populations. Such information is subject to referral bias. For example, night sweats is a symptom that has been associated in the literature with a variety of serious and unusual medical problems, including tuberculosis, autoimmune diseases, and certain malignancies (eg, lymphomas), conditions that are relatively uncommon in primary care settings. ${ }^{1}$ Questions about night sweats are frequently asked of primary care physicians by their patients. In fact, Ely et $\mathrm{al}^{2}$ reported that questions about night sweats were among the most common unanswerable clinical questions encountered in practice. Surprisingly little research, however, has been done to determine 
the incidence, prevalence, and most common causes of this symptom in primary care settings.

In our own initial investigation of this symptom, we queried 2,767 consecutive adult patients being seen by their primary care physicians. Forty-one percent reported experiencing night sweats within the previous month, including $23 \%$ with night sweats only and $18 \%$ with both night and day sweats. ${ }^{3}$ Peak prevalence in both men and women occurred between the ages of 41 and 55 years. Hot flashes were strongly associated with night sweats and with female sex, and it was difficult to separate the effects of menopause from other factors. Of the $27 \%$ of patients who were older than 64 years, $30 \%$ reported night sweats_-21\% reporting night sweats only, and $9 \%$ reporting both night and day sweats. Older patients reporting night sweats only were more likely to have insomnia, whereas those with day and night sweats had more hot flashes and were more likely to be taking corticosteroid medications.

The present study was conducted to obtain another estimate of the prevalence of night sweats, as well as day sweats and hot flashes, in a different primary care population and to identify factors associated with these symptoms as part of an effort to identify common causes. To avoid the impact of menopause, we studied older patients, using data from the first year of a longitudinal cohort study established for the study of common geriatric conditions in a primary care population.

\section{METHODS}

Individuals were eligible to participate if they were active patients (seen within 18 months) of physician members of the Oklahoma Physicians Resources/ Research Network (OKPRN) practicing within 75 miles of Oklahoma City. Participating physicians generated lists of their active patients 64 years of age and older from billing records. Patients living in a nursing home or believed by their primary care physician to be too confused to sign consent were excluded. The physicians then sent letters of invitation, and the study coordinator followed up with telephone calls. Patients who agreed to participate were sent self-administered questionnaires in the mail and were enrolled in the study at their family physicians' offices by a research nurse, who obtained formal consent and then reviewed the questionnaires for completeness. The following information was obtained from patients who delcined to participate: age, sex, level of education, self-rated health, and reasons for not participating. All patients who received questionnaires in the mail were subsequently enrolled. The questionnaire assessed demographic information, alcohol and tobacco use, a list of common symptoms, a checklist of chronic medical conditions, the Medical Outcomes Study Short Form36 (SF-36), the Quality of Well-Being Scale SelfAdministered (QWB-SA), and the Health Utility Index Mark 3 (HUI3).

The questions about night sweats, day sweats, and hot flashes were worded as follows: "During the last month, how much trouble have you had with [night sweats, excessive sweating during the daytime, hot flashes]?" Response options were no trouble, a little trouble, some trouble, a fair amount of trouble, and a great deal of trouble. The last 3 responses were considered positive, the first 2 , negative.

Other symptom questions were part of the QWBSA, SF-36, and HUI3 instruments as shown in Appendix 1, which is available online as supplemental data at http://www.annfammed.org/cgi/content/full/ 2/5/391/DC1. The QWB-SA is an 11 -item, selfadministered questionnaire scored on a scale of 0 (worst possible) to 1 (optimal) quality of life. ${ }^{4,5}$ The SF-36 is a 36 -item instrument that generates 8 different subscales, each scored from 0 (least) to 100 (most): general health, mental health, physical functioning, role-physical, bodily pain, vitality, social functioning, and role-emotional. ${ }^{6-8}$ The HUI3 is a 17 -item questionnaire that also measures health-related quality of life on a scale from 0 (lowest) to 1 (highest). ${ }^{9}$ A few additional symptom questions are also shown in Appendix 1.

A history of concurrent medical problems was elicited with a single questionnaire item: "Please indicate which of the following medical problems you have now or have experienced in the past (mark all that apply)," followed by the options listed in the Appendix.

Statistical analyses included descriptive analyses, Student's $t$ test for difference of means and chi-square for proportions, and multivariate logistic regression. Multivariate models were created to consider the relationship between dependent variables (night sweats, day sweats, and hot flashes) and factors thought to be associated based on univariate analysis $(P<.05)$. A backward elimination technique was used to identify the best model (all variables $P<.01$, and stable deviance). Age was forced into all models and removed only if $P>.01$ was a final value. Highly correlated independent variables (eg, depression, downhearted, trouble getting thoughts from mind, and inability to control life events) were initially tested by subgroups, with variables having a $P<.05$ selected for consideration in the full model. Interactions between variables that remained in the model were evaluated. Separate multivariate linear regression models were also created to consider the relationship between night sweats, day sweats, and hot flashes and scores of quality-of-life measures (HUI3, QWB-SA, SF-36). These models included sociodemographic variables (age, sex, 
education, income, and race) as covariates. Statistical analyses were performed using the Statistix 7 software program (Analytic Software, Tallahassee, Fla).

We also determined the proportions of patients with and without night sweats, day sweats, or hot flashes who had symptoms within the following subgroups: sensory impairment, musculoskeletal pain, visceral pain, and symptoms of anxiety or depression; and conversely, the proportions of patients with and without subgroup symptoms who had 1 or more of the primary symptoms We performed standard agglomerative hierarchical cluster analyses in SPSS (Statistical Package for the Social Sciences, SPSS, Inc, Chicago, Ill) with each of the primary symptoms serving as the nidus for cluster formation. Clusters were combined using squared Euclidean distance coefficients and the between-groups linkage method. Values were not standardized.

\section{RESULTS}

\section{Study Sample}

Between January 2000 and January 2001, 799 patients were enrolled from the practices of 23 primary care physicians in central Oklahoma. The physicians identified 3,979 patients aged 64 years and older from their billing records, then excluded 324 who had died, 208 who were in nursing homes, 156 who were too confused to provide reliable information, and 761 who had switched physicians (primarily because of insurance coverage). Of the remaining 2,530 eligible patients, 717 could not be reached by telephone after 3 attempts, and 1,024 declined to participate ( 87 had transportation problems, 21 had travel plans, 245 had personal or family illness, 406 lacked interest or were against research, 265 were too busy). Of 809 who agreed to participate, the project coordinator excluded 10 because they were unable to understand telephone instructions, leaving 799 who were enrolled. Four did not answer the question about night sweats and were therefore excluded from the study, leaving 795 patients. Participants were younger (mean age 73 vs 77 years, $P<.0001)$, better educated $(P<.0001)$, more likely to be male $(P=.002)$, and rated their health as better than the nonparticipants who could be reached by telephone. There was a tendency for African Americans to be more reluctant to participate $(P=.06)$. The characteristics of the study sample are shown in Table 1.

\section{Prevalence of Night Sweats, Day Sweats, and Hot Flashes}

Of the $10.3 \%$ of patients who said they were bothered to varying degrees by night sweats, $70 \%$ reported some trouble, $20 \%$ a fair amount of trouble, and $10 \%$ a great deal

\begin{tabular}{lc}
$\begin{array}{l}\text { Table 1. Characteristics of the Patient } \\
\text { Population (N = 795) }\end{array}$ \\
\hline Characteristic & Percent (n) \\
\hline Sex, female & $56.6(450)$ \\
Race & \\
White & $88.2(701)$ \\
African American & $8.8(70)$ \\
Other & $3.0(24)$ \\
Income & \\
$<\$ 15,000$ & $18.1(138)$ \\
$\$ 15,000-34,999$ & $44.4(339)$ \\
$\$ 35,000-54,999$ & $19.6(150)$ \\
$\$ 55,000-74,999$ & $10.3(79)$ \\
$>\$ 75,000$ & $7.6(58)$ \\
Education & \\
$<12$ years & $15.0(119)$ \\
HS diploma & $26.4(210)$ \\
College & $47.8(380)$ \\
Graduate degree & $10.8(86)$ \\
Cigarette use & $7.5(60)$ \\
Excessive alcohol use* & $6.3(50)$ \\
\hline
\end{tabular}

Note: mean age $=73.3$ y $(S D=5.8)$.

*Alcohol use $\geq 4$ drinks at single sitting within past 3 months.

of trouble. The corresponding percentages of the $8.7 \%$ of patients bothered by daytime sweating were $56 \%$, $33 \%$, and $11 \%$. Of the patients who were bothered by hot flashes, $62 \%$ reported some trouble, $25 \%$ a fair amount of trouble, and $13 \%$ a great deal of trouble. One hundred forty $(17.7 \%)$ patients reported at least 1 of the 3 symptoms, with $83(10.5 \%)$ reporting only $1,42(5.3 \%)$ reporting 2 , and $15(1.9 \%)$ reporting all 3 symptoms. The prevalence of night sweats, day sweats, and hot flashes by sex of the patient is displayed in Table 2 . Each of the 3 primary symptoms was associated with the others $(P<.0001)$.

\section{Variables Associated with Night Sweats, Day Sweats, and Hot Flashes}

The multivariate associations between night sweats, day sweats, and hot flashes, and relevant sociodemographic, anthropometric, and symptom variables are displayed in Table 3. Significant $(P<.01)$ associations were found between each of the primary symptoms and most other 
symptoms, with both overlap and differences between them. Variables with no univariate association with any of the 3 primary symptoms included income, marital status, having a confidant, heavy alcohol use, weight, body mass index, weight gain or loss, an expectation of worse health, loneliness, oral or dental pain, and feeling upset or blue. There were also no statistically significant univariate associations between any of the 3 primary symptoms and autoimmune diseases, sarcoidosis, tuberculosis, chronic hepatitis, cancer, thyroid problems, stroke, depression, gastroesophageal reflux disease, stomach ulcers, diverticulosis, liver disease, hypertension, osteoarthritis, osteoporosis, or Parkinson's disease.

\section{Analysis of Symptom Subgroups}

Patients who had at least 1 of the 3 primary symptoms were 1.5 times more likely to have at least 1 sensory deficit_-impaired vision, impaired hearing, impaired taste, impaired smell, or numbness on hands or feet ( $85 \%$ vs $56 \%, P<.0001)$-and 4 times more likely to have 3 or more sensory deficits $(24 \%$ vs $6 \%, P<.0001)$ than were patients with none of the primary symptoms. Conversely, those with at least 1 sensory deficit were 3.4 times more likely to have experienced any of the primary symptoms $(24 \%$ vs $7 \%, P<.0001)$.

Bodily pain (body pain, bone pain, muscle or joint pain, or hip or side pain) was experienced by $97 \%$ of those who reported night sweats, $97 \%$ of those who reported day sweats, and $95 \%$ of those with hot flashes compared with $74 \%$ of those with none of the primary symptoms $(P=.001$ or less for each comparison). Visceral pain (headache, eye pain, oral or dental pain, chest pain or pressure, stomach pain or indigestion, or genital pain) was reported by $68 \%$ of those with night sweats, $65 \%$ of those with day sweats, and $71 \%$ of those with hot flashes but by only $48 \%$ of those with none of the primary symptoms $(P=.001$ or less for each comparison). Conversely, $21 \%$ of those with bodily pain and $23 \%$ of those with visceral pain reported at least 1 of the primary symptoms compared with $4 \%(P<.0001)$ and $12 \%(P=.0003)$, respectively, of those who not report pain.

Patients with each of the 3 primary symptoms were more than twice as likely as those without those symptoms to report feeling down in the dumps, feeling anxious or fretful, or being a nervous person or having nervous spells ( $P=.001$ or less for each comparison). Collectively, one third of patients with night sweats, day sweats, and hot flashes reported being either down in the dumps or down hearted, and one half reported at least 1 of the anxiety-related symptoms. Conversely, of those who said they were down in the dumps, $34 \%$ reported at least 1 of the primary symptoms compared with $16 \%$ who said they were not down in the dumps $(P=.001)$. Twenty-eight percent of those with at least 1 anxiety symptom compared with $11 \%$ of those with no anxiety symptoms reported at least 1 of the primary symptoms $(P<.0001)$.

\begin{tabular}{|c|c|c|c|}
\hline Variable & $\begin{array}{c}\text { Night Sweats } \\
\text { OR }(95 \% \mathrm{Cl})\end{array}$ & $\begin{array}{l}\text { Day Sweats } \\
\text { OR }(95 \% \mathrm{Cl})\end{array}$ & $\begin{array}{l}\text { Hot Flashes } \\
\text { OR }(95 \% \mathrm{Cl})\end{array}$ \\
\hline Age, y & $0.94 / y(0.89-0.98)^{*}$ & - & - \\
\hline Race (nonwhite) & - & - & $3.10(1.60-598)^{\dagger}$ \\
\hline Fever & $12.60(6.58-24.14)^{\ddagger}$ & $4.10(2.14-7.87)^{\dagger}$ & $3.98(1.97-8.04)^{\ddagger}$ \\
\hline Muscle cramps & $2.84(1.53-5.24)^{+}$ & - & - \\
\hline Hearing loss & $1.84(1.03-3.27)^{\S}$ & - & - \\
\hline Vision problems & $2.45(1.41-4.27)^{*}$ & - & $2.12(1.19-3.79)^{\S}$ \\
\hline $\begin{array}{l}\text { Numbness in hands } \\
\text { and feet }\end{array}$ & $3.34(1.92-5-81)^{\ddagger}$ & - & - \\
\hline Restless legs & - & $3.22(1.76-5.89)^{\ddagger}$ & - \\
\hline Lightheadedness & - & $2.24(1.30-3.88)^{\ddagger}$ & - \\
\hline Diabetes & - & $2.19(1.22-3-92)^{*}$ & - \\
\hline Nervous spells & - & - & $1.87(1.01-3.46)^{\ddagger}$ \\
\hline Bone pain & - & - & $2.31(1.30-4.08)^{*}$ \\
\hline
\end{tabular}

Note: numbers in table are odds ratios and 95\% confidence intervals from logistic regression models.

$\mathrm{OR}=$ odds ratio; $\mathrm{Cl}=$ confidence interval.

${ }^{*} P<.01$.

$\dagger P<.001$

$\neq P<.0001$.

$\S P=.05$.

\section{Associations of Primary Symptoms With Health- Related Quality of Life}

Table 4 shows the bivariate relationships between each of the 3 primary symptoms and the various quality-of-life estimates after controlling for age, sex, race, education, and income. All 3 symptoms were associated with lower quality of life based upon at least 1 instrument. Self-rated health was negatively associated with night sweats $(P=.02)$ and hot flashes $(P=.008)$, but not day sweats.

\section{DISCUSSION}

\section{Prevalence}

Our results confirm that a significant proportion of elders experience night sweats, day sweats, and hot flashes. The $10 \%$ prevalence estimate for night sweats corre- 


\begin{tabular}{|c|c|c|c|}
\hline QOL Measure & Night Sweats & Day Sweats & Hot Flashes \\
\hline HUI3 & NS & NS & NS \\
\hline QWB-SA & $-0.045^{*}$ & NS & NS \\
\hline \multicolumn{4}{|l|}{ SF-36 } \\
\hline General health & -8.179 & $-8.262^{+}$ & $-7.006^{+}$ \\
\hline Vitality & $-9.473+$ & $-12.743^{\ddagger}$ & $-7.771+$ \\
\hline Physical functioning & $-13.338^{\ddagger}$ & $-13.368^{+}$ & $-9.672^{+}$ \\
\hline Role-physical & $-19.054^{\dagger}$ & -18.615 & NS \\
\hline Mental health & $-4.357^{*}$ & $-4.212^{*}$ & NS \\
\hline Role-emotional & $-16.682^{\ddagger}$ & $-9.892^{*}$ & NS \\
\hline Social functioning & $-6437 *$ & $-11.544+$ & NS \\
\hline Bodily pain & $-11.061^{\ddagger}$ & $-13.196^{\ddagger}$ & NS \\
\hline \multicolumn{4}{|c|}{ Note: numbers shown in table are $\beta$ coefficients from linear regression models. } \\
\hline \multicolumn{4}{|c|}{$\begin{array}{l}Q \mathrm{QL}=\text { quality of life; HUI3 = Health Utility Index Mark 3; NS = not significant; QWB-SA = Quality of } \\
\text { Well-Being Scale Self-Administered; SF-36 = Medical Outcomes Study Short Form-36. }\end{array}$} \\
\hline \multicolumn{4}{|l|}{$\begin{array}{l}* P<.05 \\
\dagger P<.001 \\
\neq P<.0001\end{array}$} \\
\hline
\end{tabular}

variables with univariate associations $(P \leq .01)$ with at least 1 of the primary symptoms, 23 (48\%) were associated with all 3 symptoms, and 33 (66\%) were associated with 2 of the 3 symptoms. Thus, all 3 primary symptoms appear to be fairly nonspecific in nature, similar to fatigue or anorexia. That none of them was associated with an expectation of worse health and only hot flashes were associated with being illness prone suggest, however, that they are not simply meaningless items in the positive review of systems. The 3 symptoms were also somewhat different. In the multivariate models, the only variables common to more than 1 of the models were fever, common to all 3 models, and impaired vision, shared by night sweats and hot flashes. sponds with a finding of an unpublished study of consecutive patients enrolled in a geriatric continuity clinic (Mold, unpublished), but it is substantially lower than the $30 \%$ reported for geriatric patients in our previously published study. ${ }^{3}$ This discrepancy may have resulted, in part, from the purposeful exclusion of patients with mild symptoms (those rating the degree of bother "a little") in the current study. If we had included them, the estimated prevalence of night sweats would have been $20 \%$. The estimates may also have been affected by the wording of the questions. In the present study, we asked how much patients were bothered by night sweats, whereas in the previous study, the question was simply "do you have night sweats?" Patient selection, however, probably accounts for most of the difference. The previous study sample comprised consecutive patients being seen in the clinic, whereas the present study involved all patients seen at least once during the past 18 months. Patients currently being seen by a physician are probably more likely to be symptomatic.

In the current study there was also some selection bias resulting from differential willingness to participate. Based upon the associations between self-rated health and both night sweats and hot flashes and the association between nonwhite race and hot flashes, we assume that this bias resulted in an underestimation of the true prevalences.

\section{Similarities and Differences Between the Primary Symptoms}

We anticipated and confirmed strong univariate associations between the 3 primary symptoms. Also, of the 50

\section{Speculations About Multivariate Associations}

Variables found to be associated with the 3 primary symptoms could represent causes, effects, or neither. In the last category are variables associated with the primary symptom through one or more confounding variables. In addition, certain variables, while not causally related, may increase awareness of, or result in greater distress from, the primary symptoms.

Fever is well known to cause sweating, so it is not surprising that fever was associated with all 3 of the primary symptoms. The association between muscle cramps and numbness and tingling of the extremities with night sweats raises questions about hyperventilation, electrolyte imbalance, and neuropathy. Alternatively, muscle cramps often occur during the night and could either cause or make one more aware of nocturnal sweating.

Anxiety could either heighten awareness or increase the degree to which one is bothered by symptoms. It could cause difficulty with sleep or result in autonomic stimulation that results in sweating. Anxiety was strongly associated with difficulty sleeping, but there was no colinearity in the multivariate model.

Sensory impairment could make one more aware of bodily sensations such as sweating, or they may be associated with mental health problems that result in night sweats. There was a strong association between visual impairment symptoms and both anxiety and depression; again, however, no colinearity could be found.

The association between day sweats and lightheadedness is interesting. Perhaps sweating results in 
chronic underhydration, and we should be as concerned about the consequences of sweating as we are about its causes. The association of day sweats with restless legs, a condition associated with iron deficiency, peripheral neuropathy, and periodic leg movements in sleep, is even less clear. The association of day sweats and diabetes may or may not be related to hypoglycemic episodes.

Patients with hot flashes were more likely to report nervous spells. This finding supports the conclusion of our previous study that hot flashes may be a symptom of panic disorder. ${ }^{3}$ The association between nonwhite race and hot flashes is interesting and unexplained. In the bivariate analyses, nonwhite race was also associated with night sweats but not day sweats. Nonwhites were also more likely to report muscle and joint pain but not visceral pain, sensory deficits, or symptoms of anxiety and depression. No interactions were found between race and any other multivariate predictors.

The lack of associations between any of the symptoms and body mass index, use of alcohol or cigarettes, and recent change in weight are interesting and probably important. Excessive weight is often blamed for daytime sweating. It is also quite interesting that, although night sweats have generally been associated in the medical literature and textbooks with specific disease processes, very few common medical conditions in the long list included in this study were associated with any of the 3 symptoms. There are a variety of possible explanations. First, many of the diseases said to cause night sweats are uncommon among primary care patients, though 50 patients in our population reported having an autoimmune disease. More importantly, the diseases reported by our patients had, no doubt, been treated with substantial resolution of symptoms. In addition, the information we obtained about diseases was by self-report and may not have been completely accurate.

Although one reason for focusing on the elderly was to eliminate menopause as an etiologic factor, even in this age-group, women were more likely than men to report hot flashes. Because we did not collect information on the use or recent discontinuation of hormone replacement therapy, we cannot be certain that estrogen deficiency was not a factor, but we doubt that it was a very important one. It may be that hot flashes as a symptom are more familiar and acceptable to women and therefore more likely to be noticed and reported by them.

\section{Strengths and Weaknesses of the Study}

This study has several strengths. It is the largest study to date of the prevalence of and factors associated with night sweats. A large number of variables were available for analysis. Associations of the 3 primary symptoms with various measures of health-related quality of life are potentially important findings that have not previously been reported.

Cross-sectional studies are, by design, exploratory. This initial approach is appropriate when little is known about the causes and consequences of the variables of interest, as in this case. Also, despite the large population size, the large number of variables considered could have resulted in some statistically significant associations occurring by chance, though by focusing primarily on those associations that have a $P<.01$, we have reduced that possibility.

No objective clinical data were available to confirm the diseases reported. Medications were not evaluated in this study. Several medications were found to be associated with night sweats in the previous study, including antidepressants, antihistamines, xanthines, and corticosteroids. The relationship between night sweats and antidepressants may actually be the result of an association between night sweats and depression, particularly as night sweats were reported with several different classes of antidepressants. A variety of medications cause diaphoresis as a side effect and should always be considered among the possible causes for night sweats and day sweats.

Although we cannot rule out an effect of climate on our results, patients were enrolled throughout the year. In our previous study, the prevalence of night sweats was the same in winter and summer. In that study, however, the question specifically excluded environmental causes. ${ }^{3}$

Higher rates of participation by younger, healthier patients and by men may have reduced our ability to detect associations between the 3 symptoms and chronic illnesses by reducing the number of participants with such illnesses. Because there was no mention of the symptoms of interest during recruitment or enrollment, it is unlikely that participants were influenced to enroll based upon symptom presence or absence.

Future studies of these symptoms should use more robust designs (case-control or cohort) and focus on the associations between each of the 3 symptoms and specific mental illnesses, sensory impairments, pain syndromes, sleep disorders, medical problems, and medications. These studies should also assess the impact of the symptoms and their causes on prognosis.

\section{CONCLUSIONS}

Night sweats, day sweats, and hot flashes are common symptoms in elderly primary care patients. Presumably related to autonomic reactions to emotional or physi- 
cal distress, they are, no doubt, more noticeable and distressing to some than to others. Though relatively nonspecific, their presence should probably prompt additional questions about febrile illnesses, diabetes, anxiety, depression, somatic and visceral pain, sensory deficits, and restless legs syndrome in addition to standard, recommended evaluations for uncommon serious diseases, such as tuberculosis and malignancies. ${ }^{10}$

To read or post commentaries in response to this article, see it online at http://www.annfammed.org/cgi/content/full/2/5/391.

Key words: Night sweats; research design; practice-based research; aged; primary health care; hot flashes/epidemiology; quality of life

Submitted August 26, 2002; submitted, revised, June 6, 2003; accepted July 20, 2003.

A version of this paper was presented at the summer OKPRN Convocation, August 17-18, 2002, Quartz Mountain Resort, Lone Wolf, Okla.

Funding support: This study was supported by a grant from the Presbyterian Health Foundation.

\section{Participating Oklahoma Physicians Resources/Research Network}

(OKPRN) practices: Oklahoma West Physicians Group, Weatherford; Family Medicine Center, Oklahoma City; Great Plains Family Practice Residency Program, Oklahoma City; Putnam North Medical Center, Oklahoma City; Westbrook Family Physicians, Edmond; Canyon Park Family Physicians, Edmond; Mid-Del Family Physicians, Midwest City; Clinton Strong, MD, El Reno; Kyle Waugh, MD, Weatherford; and the Citizens Potawatomi Clinic, Shawnee.

\section{References}

1. Mold JW, Mathew MK, Belgore S, DeHaven M. Prevalence of night sweats in primary care patients. Table W1. J Fam Pract [serial online] Available at: http://www.jfponline.com/content/2002/05/jfp_0502_ 00452.asp.

2. Ely JW, Osheroff JA, Ferguson KJ, Chambliss ML, Vinson DC, Moore JL. Lifelong self-directed learning using a computer database of clinical questions. J Fam Pract. 1997;45:382-388.

3. Mold JW, Mathew MK, Belgore S, DeHaven M. Prevalence of night sweats in primary care patients. J Fam Pract. 2002;51:452-456.

4. Kaplan RM, Sieber WJ, Ganiats TG. Comparison of the quality of well-being scale with a self-administered questionnaire. Psychol Health. 1997;12:783-791.

5. Andresen EM, Rothenberg BM, Kaplan RM. Performance of selfadministered mailed version of the quality of well-being (QWB-SA) questionnaire among older adults. Med Care. 1998;36:1349-1360.

6. Ware JE, Snow KK, Kosinski M, Gandek B. SF-36 Health Survey Manual and Interpretation Guide. Boston: New England Medical Center, The Health Institute; 1993.

7. Andresen EM, Bowley N, Rothenberg BM, Panzer R, Katz P. Testretest performance of a mailed version of the medical outcomes study 36-item short-form health survey among older adults. Med Care. 1996;34:1165-1170.

8. Lyons RA, Perry HM, Littlepage BN. Evidence for the validity of the short-form 36 questionnaire (SF-36) in an elderly population. Age Ageing. 1994;23:182-184.

9. Torrance GW. Measurement of health state utilities for economic appraisal. J Health Econ. 1986;5:1-30.

10. Viera AJ, Bond MM, Yates SW. Diagnosing night sweats. Am Fam Physician. 2003;67:1019-1024. 